\section{Native Aortic Valve Endocarditis Secondary to Staphylococcus aureus Bacteremia}

Native ("without structural heart disease") aortic valve endocarditis is very rare in children [1]. We report a child who developed 'native' aortic valve endocarditis secondary to Staphylococcus aureus bacteremia.

A 7-year-old boy presented with cellulitis over the medial aspect of the left upper thigh and pyomyositis of the left vastus lateralis accompanied by severe restriction of the left hip joint. These complaints had developed over the last 12 days. MRI detected osteomyelitis of the proximal metaphysis of the left femur with mild left hip joint effusion. His $\mathrm{Hb}$ was $10.2 \mathrm{~g} / \mathrm{dL}$ and WBC count was 29,000 cells/cu.mm, with $84 \%$ polymorphs and $16 \%$ lymphocytes. Pus aspirated from the thigh abscess grew methicillin-sensitive Staphylococcus aureus.

Therapy with standard intravenous doses of ceftriaxone, gentamicin and vancomycin was started. Three days later, the children developed breathlessness, cough and left-sided precordial inspiratory chest pain. Transthoracic echocardiography showed a large $(3.1 \mathrm{x}$ $3.3 \mathrm{~cm}$ ), mobile, friable, vegetation of the right coronary cusp of a tricuspid aortic valve, an aortic para-valvular abscess $(4 \times 2.2 \mathrm{~cm})$ communicating with the aorta, moderate pericardial effusion and trivial aortic regurgitation, tricuspid regurgitation and mitral regurgitation. The child was started on digoxin, furosemide and enalapril for heart failure.

On day four of admission, the child required tube thoracotomy for right sided pneumothorax. Over the next three weeks, there was symptomatic improvement in the child's general appearance and appetite with resolution of local left thigh inflammation and heart failure. However mild fever persisted. On the 28th day of hospital stay, the child suddenly complained of palpitations. Widened pulse pressure (BP 110/40/0 $\mathrm{mmHg}$ ) was detected and intensity of the aortic regurgitation murmur had increased. Echocardiography repeated on day 29 of admission showed an aneurysm of the right sinus of Valsava and destruction of right aortic cusp by the vegetation causing severe aortic regurgitation. However, in view of the high operative risk, the cardiac surgeon deferred intervention till the completion of antibiotics [2]. Acute intractable congestive heart failure developed on day 52 of admission necessitating endotracheal intubation and cardiopulmonary resuscitation. Rupture of the sinus of Valsava aneurysm was suspected for the sudden cardiac deterioration. He eventually succumbed 55 days after admission. Parents declined consent for autopsy.

In conclusion, the development of 'native' aortic valve endocarditis in the present case was due to the overwhelming bacteremia evident by multiple sites of infection involving soft tissue, bone and chest. Other host risk factors like primary or secondary immunodeficiency and bicuspid aortic valve had been ruled out. Till date, Staphylococcus aureus native valve infective endocarditis remains a poorly understood condition [1]. Aortic valve endocarditis due to Staphylococcus aureus is at the greatest risk for periannular extension of infection [3]. Periannular extension causes annular abscesses, aneurysms or fistulous communications [3]. Clinical signs of periannular extension are non-specific, viz. persistence of fever, recurrent emboli, worsening heart failure and new pathological murmurs $[3,4]$. Therefore recognition of periannular extension of infection often gets delayed with disastrous consequences as in the present case.

Acknowledgments: Dr SN Oak, Director (Medical Education and Major Hospital, Municipal Corporation of Greater Mumbai) and Dean of Seth GS Medical College and KEM Hospital for granting permission to publish this manuscript.

\section{Sunil Karande ANd M Muranjan Department of Pediatrics, Seth GSMedical College and KEM Hospital, Parel, Mumbai, Maharashtra, India. karandesunil@yahoo.com}

\section{REFERENCES}

1. Miro JM, Anguera I, Cabell CH, Chen AY, Stafford JA, Corey GR, et al. Staphylococcus aureus native valve infective endocarditis: report of 566 episodes from the International Collaboration on Endocarditis Merged Database. Clin Infect Dis. 2005;41:507-14.

2. Anguera I, Miro JM, Cabell CH, Abrutyn E, Fowler VG Jr, Hoen B, et al. Clinical characteristics and outcome of aortic endocarditis with periannular abscess in the International Collaboration on Endocarditis Merged Database. Am J Cardiol. 2005;96:976-81.

3. Omari B, Shapiro S, Ginzton L, Robertson JM, Ward J, Nelson RJ, et al. Predictive risk factors for periannular extension of native valve endocarditis. Clinical and echocardiographic analyses. Chest. 1989;96:1273-9.

4. Kang N, Wan S, Ng CS, Underwood MJ. Periannular extension of infective endocarditis. Ann Thorac Cardiovasc Surg. 2009;15:74-81. 